\title{
Recyclages photographiques
}

\section{Muriel Berthou Crestey}

\section{OpenEdition}

\section{Journals}

Édition électronique

URL : http://journals.openedition.org/genesis/1469

DOI : 10.4000/genesis. 1469

ISSN : 2268-1590

\section{Éditeur :}

Presses universitaires de Paris Sorbonne (PUPS), Société internationale de génétique artistique littéraire et scientifique (SIGALES)

\section{Édition imprimée}

Date de publication : 15 avril 2015

Pagination : 79-87

ISBN : 978-2-84050-992-9

ISSN : 1167-5101

Référence électronique

Muriel Berthou Crestey, «Recyclages photographiques », Genesis [En ligne], 40 | 2015, mis en ligne le

28 mars 2017, consulté le 16 mai 2019. URL : http://journals.openedition.org/genesis/1469 ; DOI :

10.4000/genesis. 1469 


\title{
Recyclages photographiques
}

\author{
Muriel Berthou Crestey
}

Les musées préservent notre passé. Le recyclage préserve notre avenir.

Theodor ADORNO

$\mathrm{B}$ ien qu'il désigne un procédé ancien, le mot « recyclage » apparaît seulement dans la langue française en 1959. Cette époque qui voit l'essor de la société de consommation et de l'obsolescence programmée déclenche un phénomène de réutilisation des matériaux. Cette pratique migre simultanément dans le domaine artistique. Le terme de « recyclage » devient un concept fondateur pour comprendre les dispositifs de création contemporains. Son introduction dans le domaine photographique suppose de redéfinir ses contours et de l'envisager comme une étape de la procédure artistique. L'observation des démarches menant à la fabrication d'images inédites issues de photos déjà existantes permet de définir un cas particulier d'étude généticophotographique qui met au jour de nouveaux types de processus créatifs. Le recyclage est à la fois une méthode de récupération et un dispositif de transformation. Dans le domaine photographique il est inducteur d'invention formelle et théorique. En altérant les modus operandi, il change le regard. C'est par cette faculté évolutive qu'il se démarque des procédés de répétition, de mimesis ${ }^{1}$, de copie $^{2}$ ou de reproduction.

De nos jours, tout est potentiellement « recyclable » (cartes postales, albums de famille ou clichés publicitaires, images de magazine, œuvres, photographies d'artistes, icônes...). Les photographes s'emparent des images préexistantes pour des raisons variées (hommage ou critique, moyen d'expérimentation formelle...). Il s'agit de définir la nature (endogène ou exogène) et les critères (techniques, théoriques ou matériels) du recyclage photographique. Nous verrons que les auteurs inscrivent ce médium non seulement dans un temps linéaire (de la prise de vue au tirage), mais aussi dans une temporalité circulaire, redonnant potentiellement une infinité d'existences aux images. À l'instar de toute photographie, l'acte ne se limite pas au temps de la prise de vue : appuyer sur le déclencheur est le fruit d'une préméditation et ce geste est suivi de quantité d'autres opérations. Le recyclage étend considérablement cet au-delà.

\section{Juxtapositions, fragmentations et effets de boucles : recyclage des fichiers numériques}

Le concept de « recyclage photographique » acquiert une densité nouvelle à partir des années 2000. À l'heure des nouvelles technologies, le recyclage est devenu un outil de création de plus en plus répandu, posant des questions éthiques et juridiques en lien avec les pratiques de réappropriation. Lorsque le couple de photographes Inez van Lamsweerde et Vinoodh Matadin choisit de recomposer l'image d'un être humain « idéal » à partir de morceaux de corps prélevés çà et là dans les images de mode qu'ils ont réalisées précédemment, la représentation de ce personnage mutant apparaît monstrueuse. Pourtant, vu séparément, chaque fragment de corps semble synonyme de perfection. Avec le logiciel Photoshop, les artistes déforment en partie la morphologie initiale du modèle selon les stéréotypes de l'imagerie des magazines, en allongeant la colonne vertébrale, comme le faisait Ingres pour d'autres raisons en peignant

1. Voir Monique Sicard, «Entre art et science, la photographie », dans Jean-Paul Fourmentraux (dir.), Art et science. Les Essentiels d'Hermès, Paris, CNRS Éditions, 2012.

2. Voir Régis Debray, Louise Merzeau (dir.), « Copie, modes d'emploi », Médium, n 32-33, 2012. 
L'Odalisque. Le recyclage sous forme de juxtapositions d'images aboutit à une iconographie intrigante par l'effet de l'ensemble ainsi produit. La photographie finale n'a plus rien à voir avec l'esthétique des corps sur lesquels chaque morceau a été prélevé. L'illusion est parfaite grâce aux logiciels de retouche. Dans ce cas, le recyclage est littéral puisque la reprise d'images implique la création d'une forme nouvelle. À l'instar des processus d'écriture utilisant le recyclage, il implique une « valeur ajoutée 3 ». Il interroge ses dispositifs et son champ d'application.

Les genres photographiques se confondent dès lors que l'on s'intéresse aux modes de fabrication des œuvres et à leur matérialité. Cette porosité des domaines découle en partie de l'abondance et du déferlement d'images déposées sur Internet. Les artistes piochent aisément dans cette matière, ce vivier iconographique. Être photographe ne signifie pas forcément « utiliser un appareil photo ». Plusieurs procédures permettent de s'approprier une représentation. Elles supposent un élargissement théorique de l'acte de création, allant de l'idée au croquis éventuel jusqu'à la mise en scène ou l'installation qui précèdent le déclic, en passant par les recadrages, photomontages, recollages, reprises d'images, tirages et manipulations de tout ordre. Les usages de l'image diffèrent également en fonction des technologies employées. Les smartphones, équipés de caméra frontale, permettent désormais de réaliser des autoportraits souvent diffusés sur les réseaux sociaux. La prolifération du selfie modifie, au $\mathrm{XxI}^{\mathrm{e}}$ siècle, les pratiques photographiques. Face à cette banque d'images vernaculaires interviennent de nouveaux usages dans le registre artistique; il y a des photographes qui font leur matériau de cette profusion d'images libres d'accès sur Internet et d'autres qui puisent dans leurs archives personnelles pour réactiver le potentiel d'anciennes images.

\section{Autoplagiat : recyclage des données}

\section{Première étape : reprise formelle (1990-2008)}

En 1990, le photographe Éric Rondepierre réalise la série des Excédents (fig. 1). En actionnant la touche «stop » du magnétoscope, le photographe prélève des photogrammes à partir des textes qui apparaissent dans les films muets qu'il visionne. Ce sont des phrases lapidaires. Elles sont inscrites en blanc sur le monochrome noir du carton. Ce processus de reprises d'images ne constitue une forme de recyclage qu'à partir du moment où il réinvestit cette imagerie dans une autre série, pour modifier son message. Concrètement, en 2008, l'artiste reprend cette image pour la réinsérer dans une autre œuvre, Arkadin (fig. 2). Elle appartient à la série des Seuils dans laquelle il recycle des images créées dans le passé, multipliant les strates temporelles. Pour retracer la genèse de cette photographie qui fonctionne sur le mode de la récupération, il faut retracer l'origine de tous les détails qui la construisent. Du point de vue de la composition plastique, une chambre d'hôtel sert de décor à ce voyage. Au premier plan figurent des images prises par l'artiste (les lunettes et l'ordinateur sont prélevés dans la première série photographique) ; au second, on aperçoit l'image d'une femme de dos, sur le départ. Il est important d'identifier la provenance de chaque fragment afin de distinguer le mode opératoire choisi pour la construction de l'image. Les différents registres (réaliste/fictionnel, passé/présent) et médiums (photographie/écriture/cinéma) s'interpénètrent puisque l'artiste fusionne les différents clichés pris dans le répertoire cinématographique et dans sa propre vie en une seule vue, rassemblant toutes les strates de temps. Les dispositifs numériques lui permettent de « recycler » ces iconographies découvertes plusieurs années auparavant. Sur le mode du « copier-coller », il injecte le passé dans le présent, actualise un instant aperçu il y a longtemps. À son tour recyclé dans une nouvelle œuvre, il fait l'effet d'une image subliminale venue contaminer le régime des représentations contemporaines. Le photographe produit une citation de son propre travail. L'artiste rend les images anonymes, sur le principe d'Internet où elles circulent souvent librement, indépendamment de toute mention indiquant leur source (elles ne sont parfois pas légendées). Le plagiat ${ }^{4}$ devient une forme d'emprunt généralisée. Appliquée à soi-même, elle forme une boucle autoréférentielle. Le recours aux logiciels de retouche lui

3. Dirk Van Hulle, « Économie textuelle : recyclage chez Proust, Mann et Joyce », Genesis, n 18, 2002, p. 91-104.

4. Hélène Maurel-Indart, Du plagiat, Paris, Gallimard, 2011 ; édition augmentée et actualisée de Du plagiat (PUF, 1999). 


\section{Quot?}

Fig. 1 : Éric Rondepierre, Chronique d'un amour (Excédents), tirage argentique sur aluminium, $80 \times 120$ cm, 1990 (C) ADAGP

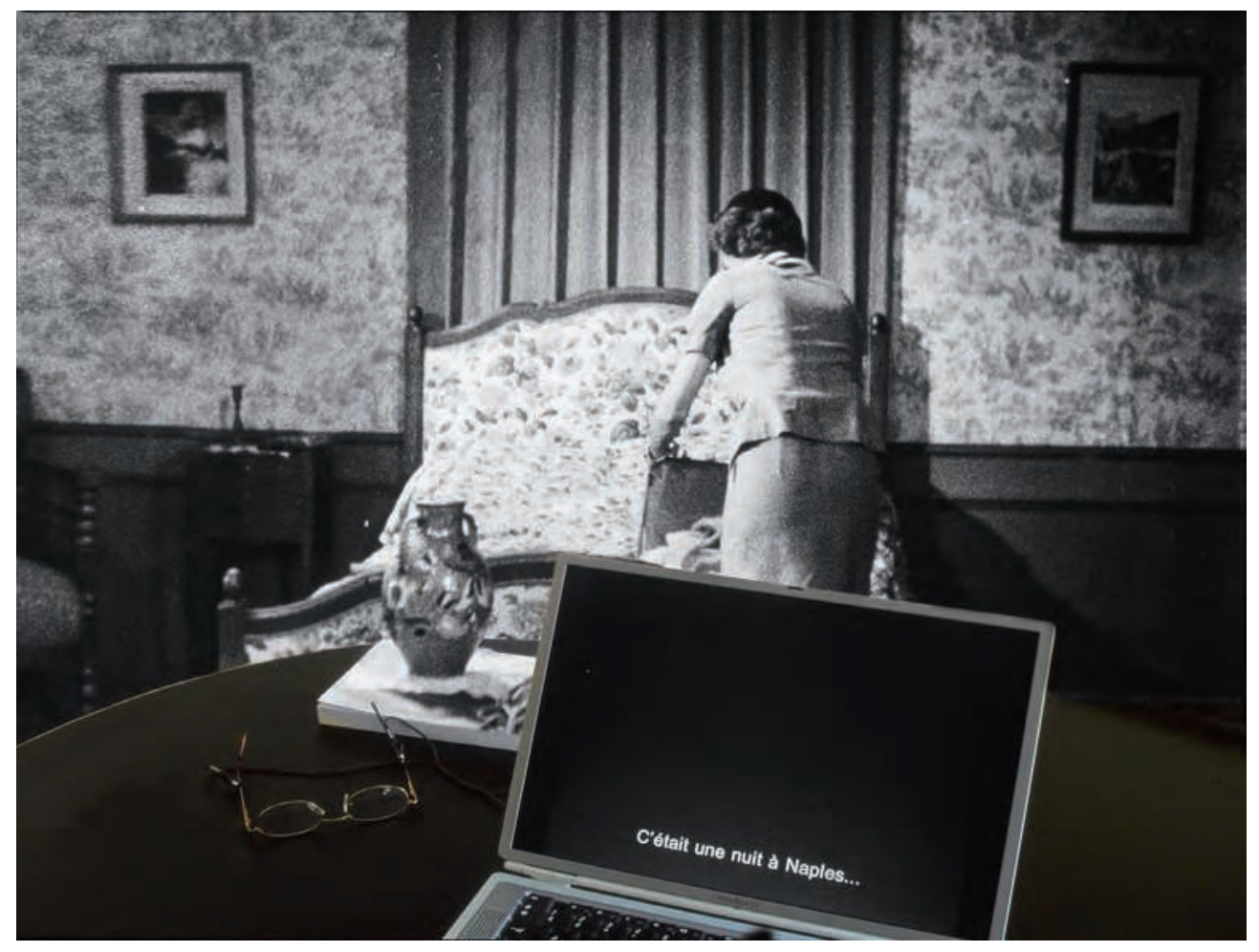

Fig. 2 : Éric Rondepierre, Arkadin (Seuils), Ilfochrome sur aluminium, $50 \times 67$ cm, 2008 (C) ADAGP 
permet toute liberté. Il transforme le texte d'origine. Éric Rondepierre reprend ce principe formel pour intégrer une phrase - « C'était une nuit à Naples... » - sur l'écran de l'ordinateur, au premier plan de l'image. Il s'agit de porter un nouvel éclairage soutenu par un contexte inédit, là où « voir, c'est toujours obtenir un double 5 ».

\section{Deuxième étape : réinvestissement et évolution du titre}

La prise en compte de l'histoire de la photographie et du cinéma constitue un paramètre important du dossier de genèse. Pour cette œuvre jouant sur les effets d'appropriation et de mise en abyme, Éric Rondepierre a recyclé le titre d'un film d'Orson Welles. À l'origine, le scénario de Welles était intitulé «Mascarade, jeu de masques »... Il est né d'une commande pour la radio de la BBC en 1951. C'est lors du tournage - en 1954 - qu'il change de nom : sur les ardoises de claps, les rushes et les photos de tournage figure la mention à M. Arkadin. C'est à cette seconde étape que Rondepierre fait allusion lorsqu'il récupère cette légende. Enfin, le film sortira en 1956 sous un autre nom Confidential report - sous la pression du producteur et du distributeur. Dans un système d'enchâssement, l'intrigue du film évoque déjà un retour vers le passé sous la forme d'un espionnage. Le photographe l'extrapole et la déploie sur un plan formel. Ici, le processus de reprises d'images ne se limite pas à un système autotélique, mais il génère une forme d'invention, à condition de retrouver toutes les citations à partir desquelles elle se construit. L'image d'origine n'apparaît plus que sous une forme métamorphosée. Cette œuvre photographique donne à voir le phénomène de déplacement, de transposition, de mouvement. Encore fautil connaître l'ordre de toutes les étapes de création pour élaborer son analyse complète.

\section{Troisième étape : l'hybridation des techniques}

Les jeux de reprises et de citation ne deviennent principe de recyclage qu'à partir du moment où ils sont vecteurs de transformation. Ce ne sont pas de simples motifs à reprendre. Dans Arkadin (2008), la référence aux Excédents intervient formellement, avec la reprise d'un détail ; le temps de production est considérablement allongé. L'œuvre initiale, ainsi que tout ce qui a permis de la mettre en place, devient matériel préparatoire. Ceci a pour corollaire de prendre en compte l'hybridation des techniques employées, étant donné le temps qui sépare les deux « reprises de vue »: d'une part, l'argentique, face à l'instantanéité du magnétoscope, d'autre part, le numérique, face à l'abondance des flux et des réseaux d'images diffusées sur Internet. Éric Rondepierre a recours dans un cas à une technique analogique (enregistrement avec magnétoscope), dans l'autre à une technologie numérique (transformation avec des logiciels de retouches d'images) ; elles révèlent chaque fois des rythmes et des mécanismes propres à chaque époque, avant et après l'apparition des nouvelles technologies. L'ordinateur sur lequel s'affiche l'œuvre initiale implique de considérer également les changements de support et la diversité qui existe dans les tirages. Ce phénomène suppose à la fois de considérer la matérialité et l'auctorialité des objets photographiques. C'est ainsi que, depuis les années 2000, l'assimilation des œuvres du passé est devenue partie prenante de certains gestes créateurs. Cette forme de recyclage créatif permet de radiographier une « ère du doublon » où le référent devient l'image, interposant un filtre avec nos représentations du réel. C'est en s'emparant de réalisations issues d'autres sensibilités que des artistes parviennent à exprimer leur singularité en se réappropriant l'œuvre d'origine. La nouveauté émerge de ce qui a déjà été perçu, voire utilisé. Cette idée selon laquelle le recyclage au sens strict est une photographie à partir de déchets va être prise à la lettre par d'autres.

\section{Recyclage matériel : littéralité et littérarité}

Vik Muniz, photographe brésilien ayant grandi à São Paulo, a installé son atelier dans la décharge de Jardim Gramacho, le temps du tournage du film Waste Land (2010). Dans ce cas, l'artiste s'inspire de tableaux célèbres pour les reconstituer avec l'aide des personnes travaillant dans la décharge. Les trieurs sont devenus ses modèles. Dans un premier temps, il les photographie. Ses portraits sont projetés en grand format de façon à recréer ceux-ci à partir des matériaux glanés dans la décharge (plastiques, papiers, bandes magnétiques...). Il conçoit donc une première

5. Sarah Kofman, Camera obscura. De l'idéologie, Paris, Galilée, 1973, p. 37. 
photographie sur un mode simple, où il existe un référent (les personnes à photographier) et un objet qui en restitue l'image (le portrait photographique). Quantité de protocoles supplémentaires sont activés lorsqu'il reconstitue ces effigies à partir d'éléments disparates. Cette forme de reconversion artistique apparaît comme un véritable acte de sublimation : transformation de déchets en œuvre d'art. Une fois l'installation terminée, la photographie du résultat devient l'œuvre finale. Elle sera vendue au profit d'une association (Catadores) et offerte aux modèles qui l'accrocheront dans les favelas. L'iconographie est née du recyclage au sens premier mais aussi d'un échange, d'une relation humaine faisant de la vie des personnes un matériau au même titre que les autres. L'artiste nourrit son œuvre des rebuts pour en faire des rébus, les réinvestir dans une chaîne de signifiants et leur donner une autre fin. Cette procédure nécessite de penser la dialectique entre l'unicité et les multiples, l'identité de l'auteur face à la figure de l'interprète.

\section{Recyclage technique et technologique}

Le projet intitulé Orogenèses de Joan Fontcuberta a été élaboré à partir d'un logiciel informatique - Terragen - initialement destiné aux militaires. Il leur permet de traduire des cartes géographiques sous forme d'images de synthèse représentant le paysage en relief, à des fins de repérage avant de se rendre sur les lieux des opérations. Dans le cadre de l'utilisation première, les images qui en émanent prennent des allures archétypales et hyperréalistes. Mais Fontcuberta a complètement détourné ce dispositif qui lui a été proposé lors d'une résidence d'artiste dans les montagnes rocheuses de l'ouest du Canada, qu'il évoque par ces mots : «un paysage paradisiaque ». Il détourne ce logiciel à des fins artistiques. Au moment de la résidence, en 1992, l'objectif de devoir produire de nouveaux paysages imaginaires l'avait rendu sceptique. Il explique :

Pour moi, artiste espagnol, né à Barcelone, dans cette ville envahie par la pollution et le bruit, j'avais imaginé faire une œuvre de fiction en étant immergé pendant quelques semaines dans ce superbe paysage, premier parc national protégé du Canada. Et c'est très étrange car j'avais l'impression de m'être introduit dans le paysage d'une agence de voyage ou dans une carte postale, tellement c'était beau. C'était le paradoxe, car dans cet endroit on avait invité des artistes et des scientifiques à travailler ensemble pour produire des logiciels de réalité virtuelle et construire des paysages en trois dimensions qui n'existaient pas réellement ${ }^{6}$.

Ainsi témoigne-t-il de son étonnement face à cette demande consistant à inventer des environnements artificiels, alors même qu'à l'extérieur de sublimes panoramas rivalisaient de beauté naturelle. De retour en Espagne, réfléchissant dans son atelier sur le rôle du paysage dans l'histoire de l'art, il réinvestit ce projet laissé en attente et modifie les données à partir desquelles il faut élaborer ces nouveaux sites. Huit années se sont écoulées entre ces deux expériences.

En 2000, en écho à sa contemplation des paysages canadiens, le premier tableau choisi par Fontcuberta est Le Voyageur contemplant une mer de nuages de Friedrich. Il scanne une reproduction, l'introduit dans le logiciel qui la décrypte comme s'il s'agissait d'un document impartial et réel. Ainsi, les œuvres d'art de ses artistes phares constituent dans un premier temps la matrice, le code source. Le logiciel les transforme. Dans les Orogenèses, le logiciel ne fait aucune différence entre une simple carte et la reproduction d'un tableau. Mais il « recycle » indistinctement chaque ligne en fonction de codes préétablis. Il traduit - mécaniquement - les densités et valeurs chromatiques en termes d'altitudes. Les couleurs sont réinterprétées sous forme de volumes et se métamorphosent. L'image en deux dimensions est convertie en trois dimensions. Les correspondances ainsi générées donnent une réalité illusoire à ces paysages imaginés, en les présentant pourtant comme des reliefs existants. La confrontation entre l'original (soit la peinture) et son interprétation favorise les points de comparaison. La nature fantastique de ces territoires est issue d'un «voyage dans l'image » où l'histoire de l'art agit comme un filtre. Le moyen utilisé s'apparente à une fin. La peinture est un préalable à l'œuvre dite «photographique ». Elle devient un vivier de création migrant vers les nouvelles technologies. Le déplacement provient de la mise en abyme de la nature

6. Extrait d'un entretien avec l'artiste, 28 août 2010. 
interprétée par l'artiste puis retraduite par le logiciel, comme s'il s'agissait de données scientifiques.

L'une des caractéristiques du recyclage photographique est de favoriser le métissage des médiums ; ainsi, une photographie peut émerger d'un support pictural, à condition de retracer la chaîne des interventions et des références du photographe. La confusion entre le reportage et la fiction scelle l'origine du projet. L'orogenèse désigne la partie de la géographie concernant le mouvement de l'écorce terrestre. Ici, il s'agit d'examiner la tectonique des plaques artistiques, où les médiums se chevauchent pour révéler de nouvelles géographies. Parfois, l'artiste met ce logiciel gratuit au service du public dans les lieux d'exposition. Les spectateurs produisent leurs propres paysages, véhiculant ainsi leur vision mentale sous forme imagée. Ici, le paysage devient un autre lieu de transport, une reproduction vivante de l'œuvre d'un peintre dans laquelle on a ainsi l'illusion de pouvoir déambuler. On se promène - virtuellement - dans son imaginaire devenu réalité, imagé avec une précision photographique.

L'artiste précise que le contexte dans lequel sont présentées ses photographies est essentiel car il permet l'identification et fonde la réception du spectateur. Le cadre du musée implique par exemple une valeur testimoniale. Ainsi avait-il piégé les critiques et historiens en exposant une série composée "à la manière de Picasso » dans une institution dédiée au maître. Chacun s'était accordé à y voir des œuvres inédites alors même qu'il s'agissait d'une supercherie.

\section{Causes et effets du recyclage : pour une redéfinition des corpus}

Les reproductions iconographiques se multiplient, se superposent les unes aux autres et finissent par s'annuler à force de se recouvrir. Avec les Googlegrammes (fig. 3), l'artiste espagnol réinvestit la technique de « recyclage technologique » : il utilise un logiciel de photomosaïque pour reconstituer l'héliographie de Niépce, considérée comme la première photographie de l'histoire des représentations. En insérant les mots «photo » et « foto » dans le moteur de recherche, il obtient dix mille images recyclables.
Puis, le programme du logiciel les agence afin de recréer visuellement la forme de l'image à partir de toutes les petites vignettes rassemblées. La poïésis photographique relève de l'accumulation d'informations. Les images d'origine disparaissent dans cette "survisibilité » et cet excès de stimuli. Elles se combinent avec d'autres formes, comme un immense réseau composant des œuvres aux densités inédites. La notion d'archives est modifiée puisque le corpus d'œuvres concerné est infini ; il s'accroît quotidiennement sur la trame d'Internet. Les logiciels informatiques constituent alors un recours à la classification en instaurant de nouvelles pratiques.

Pionnier dans l'expérimentation des nouvelles technologies, Andreas Müller-Pohle va plus loin (fig. 4) dans ces effets de métamorphoses. Il s'empare lui aussi de la même héliographie de Niépce mais pour en faire une abstraction numérique, une interprétation dont seule la connaissance du protocole de réalisation permet de retracer l'image d'origine. Habitué à composer des représentations à partir de codes (génétiques, numériques, politiques), interrogeant les flux, il traduit les formes, les valeurs et les contrastes en termes numériques. Seuls des chiffres correspondent désormais à ce qui relevait du visible.

\section{Recyclage théorique et méthodologie : innovation et réactivation}

La compréhension de toute photographie découle de paramètres inscrits dans la durée et elle implique la prise en compte de phases successives. Dès lors, l'observation des actions menant à la création photographique s'avère être indispensable à la découverte des phénomènes de création ; elle s'ajoute à l'histoire de l'art et des techniques, la sémiologie, l'herméneutique et l'esthétique, ouvrant des réseaux pour saisir le sens des œuvres. Il y a un recyclage partiel des concepts qui s'adaptent progressivement à cet objet de recherche mouvant qu'est la photographie. Une image ne naît pas ex nihilo. Elle est le fruit de procédures, de protocoles, d'interventions successives qui lui confèrent une singularité. Elle nécessite d'être replacée dans la série où elle s'inscrit, par rapport à l'image qui la précède et dans l'ensemble de la carrière de l'artiste. Les processus de création qui conduisent 


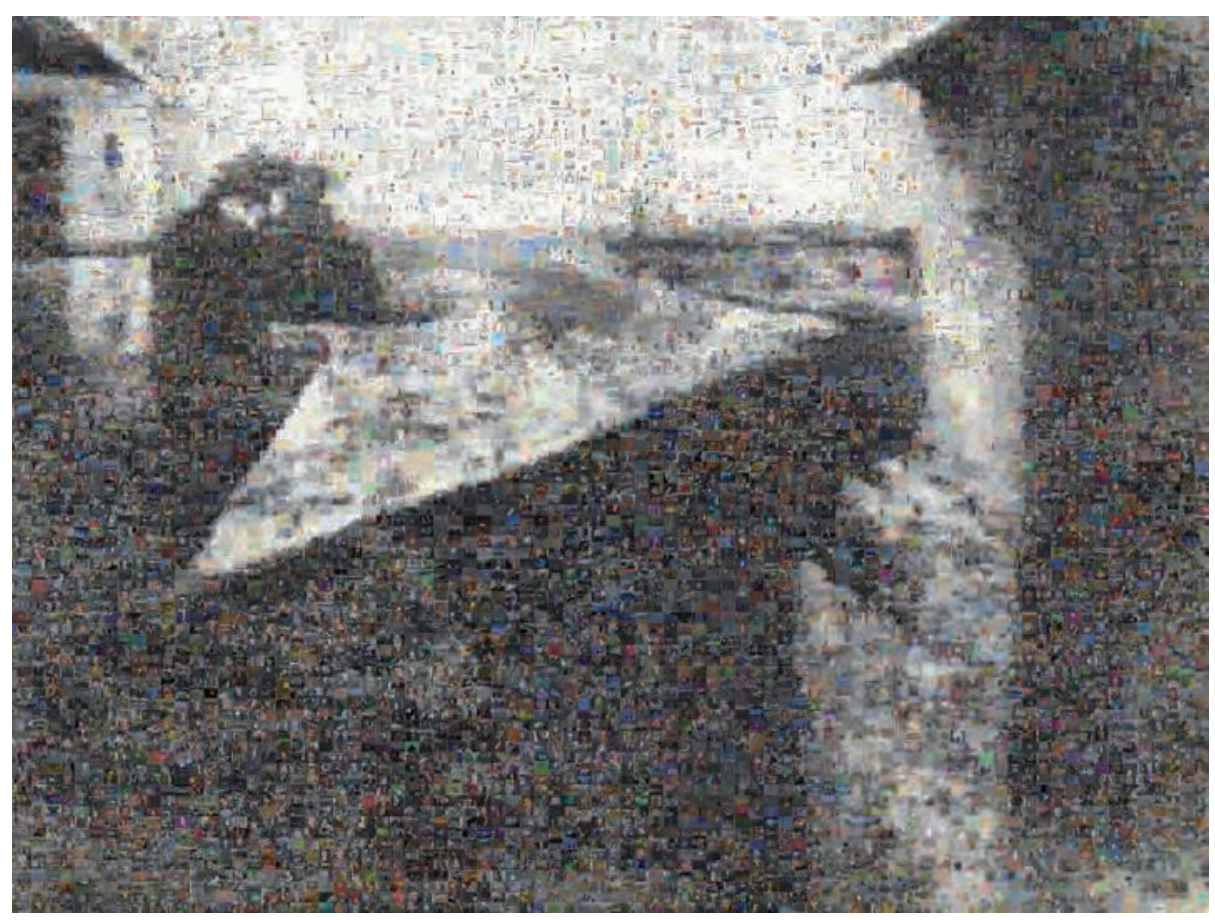

Fig. 3 : Joan Fontcuberta, Googlegrammes, Niépce, 2005 (C) Joan Fontcuberta

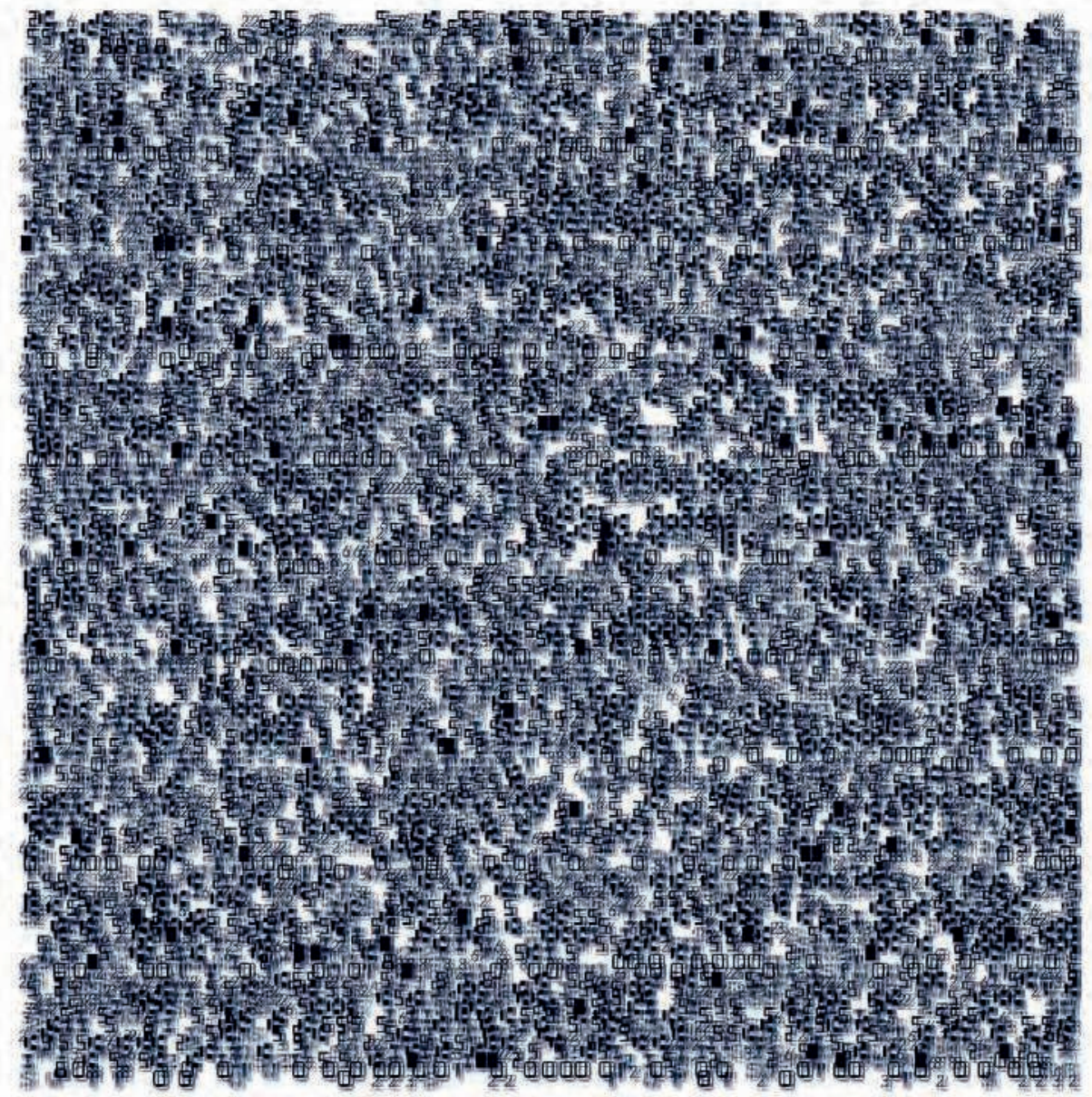

Fig. 4 : Andreas Müller-Pohle, Digital Scores IV (after Nicéphore Niépce), 1998, Iris inkjet print, $66 \times 66 \mathrm{~cm}$. Production : Artificial Image, Berlin 
les artistes à choisir des matériaux existants nécessitent pour le généticien de réinvestir des méthodologies, des matériaux et des objets heuristiques issus des sciences humaines - approche des techniques, des procédures de l'histoire et de l'anthropologie de l'art, de l'esthétique et des méthodes employées dans l'étude des manuscrits - pour les réintroduire dans une logique visuelle. Les documents qui accompagnent la gestation et la métamorphose d'une œuvre permettent alors de constituer « l'avant-image ». Ce néologisme n'a pas encore fait l'objet de véritable théorisation à ce jour. Il se construit sur le modèle de l'« avant-texte 7 » et invite à considérer les dispositifs de fabrication avec la même attention que l'œuvre qui en découle. Il s'agit de reconstituer les conditions de l'acte de création, en recherchant les matériaux qui l'ont engendré. Dans le domaine de la photographie, il existe des plans, des esquisses, des repentirs, de la documentation. Il y a une grande variété d'éléments préparatoires. La pratique du recyclage les décuple. Il faut considérer l'avant-image non comme la seule planche-contact, le seul croquis indiquant un positionnement au modèle ou la seule note d'intention de l'artiste, mais comme l'ensemble de ces documents réorganisés pour comprendre les étapes constitutives d'une œuvre photographique. Parmi les procédures privilégiées par les artistes contemporains, le recyclage représente une stratégie particulièrement significative dans l'élaboration d'une photographie. Il donne une double historicisation des processus. C'est dans le mixage des disciplines et le découpage des phases d'élaboration que naît la signification de l'œuvre et du visible. Deux attitudes se rejoignent puisqu'il faut « prendre en compte simultanément l'occasion qui surgit et celle qui recycle, interroger à ce propos la part du milieu dans la production artistique, c'est rechercher les points d'ancrage du hasard et de l'intention $[\ldots]^{8} »$. La mémoire processuelle suppose de réinvestir une chronologie dans l'analyse des images photographiques, faisant renaître des réminiscences.

\section{Hommages, greffes, cycles et renaissance}

Le recyclage repose sur des techniques variées. Tantôt, il relève de la fragmentation, de la juxtaposition ou du collage de plusieurs représentations, tantôt il fonctionne en termes d'accumulation et de sédimentation, sur le modèle du palimpseste où les couches s'accumulent. L'artiste londonien Idris Khan opte pour cette dernière méthode. Il exploite les potentialités du numérique en utilisant la superposition d'images ou de textes. Ainsi recycle-t-il par exemple l'iconographie de Bernd et Hilla Becher avec leur série photographique des Châteaux d'eau. Il place toutes les images les unes sur les autres. Dans sa série intitulée Chaque..., la multiplication des strates produit simultanément un effacement et une révélation. Elles fusionnent en un seul agrégat; les trames enchevêtrées et illisibles font apparaître, comme par mirage, les lignes communes entre les différentes photographies d'origine. Le photographe nous donne à percevoir la vibration d'une idée encore vivante, reformulée visuellement, en deçà de sa compréhension littérale.

Le concept de recyclage est un outil théorique pour penser certains processus photographiques et l'importance des phases préparatoires de ce médium. La clé pour appréhender ce phénomène particulièrement prégnant à l'aune des nouvelles technologies réside dans l'équilibre à trouver entre la figuration du détail et l'image d'ensemble, la reprise d'images et leurs réinterprétations. La récupération insère l'archive au cœur de la création photographique. Elle peut être de deux ordres : générique et publique (Internet) ou intimiste et privée (dans le cas des fonds personnels classés par leurs auteurs).

Considérées dans leurs états successifs, les photographies dévoilent leur mécanisme et leur caractère vivant, mouvant. Le médium photographique n'est plus seulement le lieu du figé, mais devient le lieu de l'acte en puissance et du processuel.

7. Si l'on se réfère à la définition qu'en donne Daniel Ferrer dans le dictionnaire de génétique littéraire disponible en ligne sur le site de l'ITEM (<www.item.ens.fr/index.php?id=577463>, l'avant-texte désigne en premier lieu la « reconstitution critique de ce qui a précédé un texte » et « l'ensemble virtuel des documents de genèse d'une œuvre ou d'un projet d'œuvre ». Ce terme est introduit par Jean Bellemin-Noël en 1972 dans son ouvrage fondateur: Le Texte et l'avant-texte : les brouillons d'un poème de Milosz (Paris, Larousse).

8. Monique Sicard, «L'occasion qui surgit, l'occasion qui recycle », dans Michel Bouvier, Roger-Yves Roche, Évelyne Rogniat (dir.), Arts d'occasion. Photographie et cinéma, Lyon, PUL, 2001, p. 50. 
Muriel Berthou Crestey est Docteur en esthétique, qualifiée MCF (18e section, CNU) et chercheur contractuel à l'ITEM (CNRS-ENS), membre des programmes ANR «Photopaysage », «Flaubert et le pouvoir des images ». Spécialiste de photographie, lauréate de la bourse de recherche du Centre national des arts plastiques (2012), elle a collaboré au Dictionnaire de la photographie (Thames \& Hudson) et à l'Allgemeines Künstlerlexikon (De Gruyter), menant des recherches pluridisciplinaires sur les processus de création.

murielberthoucrestey@gmail.com

\section{Résumés Recyclages photographiques}

En quoi les processus de reprises d'images peuvent-ils générer des formes d'invention en photographie ? L'étude du phénomène de « recyclage photographique » suppose de réaliser un dossier de genèse en repérant les phases de création qui répondent à des protocoles variés : recadrages, photomontages, recollages, reprises ou accumulation d'images et manipulations dont il s'agit de retracer la chronologie. Les raisons de ces réinvestissements sont multiples (hommage, critique, mode d'expérimentation plastique...). La nature des images reprises est variable (carte postale, image vernaculaire ou chefd'œuvre). Les moyens utilisés permettent d'aborder différentes procédures, allant du « copier-coller » à l' « autoplagiat », en passant par la juxtaposition d'images, le recyclage technique, technologique, matériel et littéral, voire théorique. Les archives deviennent un vivier créatif. Ces stratagèmes ouvrent la voie à des modes opératoires de déplacement, de citation, de transposition et de passages successifs d'un référent à l'autre.

How can the processes of image-repeat generate inventive photographic forms? Studying the phenomenon of "photographic recycling" implies producing a genetic "dossier" by identifying the successive creative stages that apply various protocols: cropping, photomontage, snipping, image-repeat or image accumulation and manipulation, for each of which the chronology needs to be ascertained. There are many reasons for this reinvestment (tribute, criticism, plastic arts experiments...). The types of the image-repeat are variable (postcard, vernacular image or masterpiece). The means employed make it possible to use various procedures: copy-paste, self-plagiarism, image juxtaposition, technical, material and literal or even theoretical recycling. Archives become a creative pool. These devices make way for operative modes of displacement, quotation and transposition of successive passages from one referent to another.

Inwiefern können die Prozesse der Wiederholung von Bildern Weisen der Erfindung in der Photographie generieren? Die Studie über das Phänomen des ,photographischen Recyling“" zielt darauf ab, ein „dossier génétique“ herzustellen, indem sie die Phasen des Schaffens ausfindig macht, die auf verschiedene Protokolle antworten: Recadrage, Photomontage, Recollage, Wiederholungen oder Anhäufung von Bildern, wobei es darum geht, die Chronologie nachzuzeichnen. Die Gründe für diese Wiederaufnahme sind vielfältig (Hommage, Kritik, Versuch der plastischen Gestaltung ...). Die Natur der wiederholten Bilder ist unterschiedlich (Postkarte, Amateurbild oder Meisterwerk). Die verwendeten Mittel erlauben verschiedene Verfahren: Copy \& Paste, Selbstplagiat, Aneinanderreihung von Bildern sowie verschiedene Arten des Recyclings (technisch, technologisch, materiell oder sprachlich, d.h. theoretisch). Das Archiv wird eine kreative Werkstatt. Die Strategeme ebnen den Weg für funktionelle Weisen des Ersetzens, des Zitierens, der Transposition und der sukzessiven Übergänge von einem Bezugspunkt zum anderen.
¿En qué medida la reutilización de imágenes puede generar formas de invención en fotografía? El estudio del fenómeno del "reciclado fotográfico" exige la realización de un dossier de génesis que detecte las fases de creación que corresponden a protocolos variados: reenfoque, fotomontaje, collage, reposición o acumulación de imágenes y manipulaciones, cuya cronología requiere ser establecida. Los motivos de esas reutilizaciones son múltiples: homenaje, crítica, modo de experimentación plástica, etc.; la naturaleza de las imágenes reutilizadas es variada: tarjeta postal, imagen vernácula u obra maestra. Los medios utilizados permiten abordar diferentes procedimientos, que van desde el "copiar-pegar" hasta el "autoplagio", pasando por la yuxtaposición de imágenes, el reciclado técnico, tecnológico, material y literal, e incluso teórico. Los archivos se convierten en un semillero creativo. Estas estratagemas permiten acceder a modos operatorios de desplazamiento, cita, transposición y pasajes sucesivos de un referente a otro.

In che cosa i processi di recupero delle immagini possono generare forme d'invenzione in fotografia? Lo studio del fenomeno del "riciclaggio fotografico" presuppone la realizzazione di un dossier genetico che rintracci le fasi della creazione rispondenti a diversi protocolli: re-inquadrature, fotomontaggi, collage, riutilizzazioni o accumulazioni d'immagini e manipolazioni di cui bisogna ripercorrere la cronologia. Le ragioni di questi reimpieghi sono diverse (omaggio, critica, sperimentazione plastica...) e la natura delle immagini riprese è varia (cartolina, immagine banale o capolavoro). I mezzi utilizzati permettono di esaminare diverse procedure che vanno dal "copia-incolla" all'"auto plagio", passando dalla giustapposizione d'immagini, il riciclaggio tecnico, tecnologico, materiale, letterale e perfino teorico. Gli archivi diventano un vivaio creativo. Questi stratagemmi aprono la via a modi operativi di spostamento, citazione, trasposizione e passaggi successivi da un referente all'altro.

$\mathbf{E}_{\mathrm{m}}$ que medida os processos de reprodução de imagens podem gerar novas formas de invenção em fotografia? O estudo do fenómeno da "reciclagem fotográfica" parte de um dossier genético que identifica fases criativas que obedecem a variados protocolos: reenquadramento, fotomontagem, recolagem, reprodução, acumulação ou manipulação de imagens, cuja genealogia tem de ser reencontrada. As razões para estas operações são múltiplas (tributo, crítica, experimentação plástica...) A natureza das imagens recicladas é variável (cartão postal, imagem primitiva, obra de arte). Os procedimentos também são diferentes, variando entre o corte-e-cola e o auto-plágio, passando pela justaposição de imagens, a reciclagem técnica, tecnológica, material e literal, mesmo teórica. Os arquivos convertem-se em viveiros criativos. Estes estratagemas abrem caminho a operações de deslocação, transferência, citação e intercâmbio entre referentes. 